\section{Socioeconomic, demographic and lifestyle factors associated with dietary patterns of women living in Southern Brazil}

\author{
Fatores sócio-econômicos, demográficos e de \\ estilo de vida associados com padrões alimentares \\ de mulheres residentes no Sul do Brasil
}

\author{
1 Programa de Pós-gradu- \\ ação em Saúde Coletiva \\ Universidade do Vale do Rio \\ dos Sinos, \\ São Leopoldo, Brasil. \\ 2 St. Michael's Hospital, \\ University of Toronto, \\ Toronto, Canada. \\ Correspondence \\ M. T. A. Olinto \\ Programa de Pós-gradu- \\ ação em Saúde Coletiva \\ Universidade do Vale do \\ Rio dos Sinos. \\ Av. Unisinos 950 \\ São Leopoldo, RS \\ 91022-000, Brasil. \\ mtolinto@unisinos.br
}

\begin{abstract}
This study focused on the association between dietary patterns and socio-demographic and lifestyle factors. A population-based crosssectional study was performed in 1,026 adult women from Southern Brazil. Dietary patterns were identified using principal components analysis. In the multivariable analysis, a Poisson regression model was used to estimate the prevalence ratio and 95\%CI. Wealthier women were more likely to follow healthy diets. A Low Cost Healthy Diet was more prevalent among women with a partner, and a High Cost Healthy Diet was more prevalent among women not currently working and who exercised regularly. Women with lower education levels were less likely to follow a Low Cost Healthy Diet, but more likely to follow the Higher-Risk Low Cost Diet. The Low Cost Higher-Risk Diet was more prevalent among women with a lower income. Low and medium cost healthy diets were positively associated with age. The results showed that women's dietary choices are influenced by socioeconomic factors and are not only dependent on food prices.
\end{abstract}

Food Habits; Socioeconomic Factors; Women

\author{
Adriana Lenz ${ }^{1}$ \\ Maria Teresa Anselmo Olinto ${ }^{1}$ \\ Juvenal S. Dias-da-Costa ${ }^{1}$ \\ Ana Luisa Alves 1 \\ Marcos Balbinotti 1 \\ Marcos Pascoal Pattussi 1 \\ Diego Garcia Bassani 2
}

\section{Introduction}

Investigating the dietary patterns of specific population groups can improve our understanding of the complex relationship between diet and health. The identification of factors that are associated with dietary patterns represents a useful indicator of health and may help to guide policy making processes that promote an affordable, healthy diet $1,2,3$.

Dietary patterns in a population are influenced by biological, nutritional, socioeconomic, and demographic characteristics. Moreover, these reflect local conditions in terms of the availability of foods and the cultural aspects of population subgroups $4,5,6,7,8$.

The evolution offood consumption patterns in large Brazilian cities points to unfavorable trends, especially from the standpoint of the association between chronic diseases with diets rich in high energy foods that have low fiber and micronutrient content and include excessively fatty foods (including those with saturated fats) ${ }^{9}$.

Dietary patterns and their determinants have been studied in several countries 10,11,12 A recent review of 93 studies conducted in diverse populations identified eating patterns using cluster and factor analyses 13. Most of those studies were undertaken in developed countries, and the patterns that emerged were labeled according to specific combinations of food and/or descriptions of nutritional compo- 
sition; however, food costs were not considered when labeling the patterns.

The present study investigates the socioeconomic, demographic and lifestyle factors that are associated with dietary patterns in Brazilian women. This study is innovative in that it labels dietary patterns according to their cost and relationship with disease risk.

\section{Methods}

We carried out a population-based cross-sectional study in 2003, with a representative sample of women aged between 20 and 60, living in an urban area of São Leopoldo, a mid-size city located in Southern Brazil. The sample size was calculated to identify an odds ratio of 2.0 with a $95 \%$ confidence interval and $80 \%$ statistical power, assuming a ratio between unexposed and exposed of 1:3 according to the socioeconomic distribution. In order to compensate for potential losses/refusals during fieldwork and for confounder control, we increased the estimated required sample size by $25 \%$. Sample selection was carried out in multiple stages. Initially, we systematically selected 40 census sectors from the 270 sectors in the municipality. Within each of these sectors, we randomly selected blocks and households. All 1,084 women aged 20-60 living in the selected households were invited to participate in the study.

Standardized, pre-coded, pre-tested questionnaires were administered by trained interviewers. The following demographic and socioeconomic variables were included: age, categorized into 10-year groups; skin color, observed by the interviewer and categorized as white or nonwhite; marital status, reported by the subject as with or without a partner; employment status, categorized as working or not working at the time of the study; economic class, according to the classification of the Brazilian Association of Research Institutes (ABEP), divided into A, B, $\mathrm{C}, \mathrm{D}$, and $\mathrm{E}$, with $\mathrm{A}$ being the highest economic class, considering both household assets (i.e. car, television, washing machine, etc.) and the education level of the head of the household 14; per capita family income, based on the reported income for each family member in the last month and categorized according to quartiles of minimum wages per capita; and education, in years of study, categorized according to the ABEP classification. Physical activity was classified using a version of an instrument designed for the Brazilian Program for Physical Activity, which classifies subjects as sedentary or non-sedentary 15 . Subjects were classified as sedentary when reporting no physical activity, no physical activity during leisure time, or light physical activity only once a week. All other women were classified as nonsedentary. Alcohol consumption was quantified in grams of ethanol per day and categorized using a cutoff of $30 \mathrm{~g} /$ day 16 .

A Food Frequency Questionnaire (FFQ) comprising 70 food items was used to identify dietary patterns. In the month preceding the interview, the frequency of food consumption, but not the amount, was recorded. This questionnaire was designed based on a previous questionnaire used in a similar area, and was adapted following a pilot study. All 70 food items were used in the analysis, i.e. food items were not grouped. Principal components analysis with orthogonal transformation (varimax) was used to identify food patterns. The results of the Kaiser-Meyer-Olkin (KMO) test and of Bartlett's test of sphericity supported the use of a factorial matrix ${ }^{17}$. The number of retained factors was based on the following criteria: components with an eigenvalue $>1$, scree plot test and interpretability of the factors. Food items were considered to load on a factor if they had a correlation $\geq 0.30$.

Five dietary patterns were identified each consisting of 10 items (foods). 20 items were excluded because of a weak or an inverse correlation in the patterns. This procedure was described in detail previously 17 . These patterns were named according to whether they were likely to confer protection from or increase the risk of chronic diseases 18,19 and according to cost (low, medium, and high cost). We identified three healthy dietary patterns and two higher risk dietary patterns: healthy diet-low cost (HLC), healthy diet-medium cost (HMC), healthy diet-high cost (HHC), higher-risk diet-low cost (RLC), and higher-risk diet-high cost (RHC). The dietary pattern costs were determined according to the prices of the standard serving 20 using prices taken from the largest food market in the area 17. Table 1 presents the components and costs of each dietary pattern. The scores for dietary patterns ranged from 10 (lowest consumption frequency) to 58 (highest consumption frequency).

For bivariate analysis, each of the five dietary patterns was categorized into a dichotomous variable related to high consumption of a given pattern: yes or no, using the upper quartile as a cut-off point for high consumption. Bivariate analyses included chi-squared tests and prevalence ratios with respective $95 \%$ confidence intervals. Only variables with significance below 0.20 in the bivariate analysis were included in the multivariate analysis. Multivariate analysis was based on an a priori conceptual framework 21. Two blocks were incorporated into the model. A 
distal block included socioeconomic and demographic variables, and the proximal block incorporated behavioral variables. In order for a variable to be considered as a potential confounder and to be retained in the multivariate model, it had to show a $\mathrm{p}$ value $<0.10$ in the likelihood ratio test. Finally, variables were considered to be significantly associated with the outcome for $\mathrm{p}$ value $\leq 0.05$ in the likelihood ratio test.

The study protocol was approved by the Research Ethics Committee at the Federal University of Pelotas Medical School. Written informed consent was obtained from all participants. Confidentiality was maintained throughout the study.

\section{Results}

Of the 1,084 women from the selected households, 58 (5.4\%) could not be contacted or did not agree to participate in the study. The final sample included 1,026 women aged between 20 and 60 . Almost half of these women were aged 20-39, and the mean age was 38 . Over two-thirds of the subjects lived with a partner, $84 \%$ were white, $26 \%$ belonged to economic classes $\mathrm{D}$ and $\mathrm{E}$, almost $50 \%$ had incomes up to 1.33 times the minimum wage, and $43.4 \%$ were unemployed at the time of the interview.

With regard to behavioral variables, approximately $77.5 \%$ of the women were non-smokers (59.1\% never had smoked and $18.4 \%$ were former smokers) and $22.5 \%$ were smokers at the time of the interview; $68 \%$ did not engage in regular physical activity.

Table 1 describes each dietary pattern and the cost per serving for each pattern. Despite the similarity in terms of price per serving between the HLC and HMC, there was a large difference in mean consumption frequency: 36.4 and 16.1, respectively. Both the RLC and RHC patterns showed relatively high consumption: 37.3 and 30.1 , respectively. The cost of a RHC serving, composed of industrialized foods, was higher than all other dietary patterns.

In a bivariate analysis, the dietary patterns were treated as dichotomous variables. Healthy low- and medium-cost diets (HLC and HMC) were more frequent in women over 40 , from economic classes A and B, with a high level of education

Dietary patterns used in this study ( $n=1,026$ participants).

\begin{tabular}{|c|c|c|c|c|c|}
\hline \multirow{2}{*}{$\begin{array}{l}\text { Dietary patterns } \\
\text { (price per serving *) }\end{array}$} & \multirow[t]{2}{*}{ Item } & \multicolumn{4}{|c|}{ Frequency of consumption/month } \\
\hline & & Mean & Range & $\begin{array}{l}\text { Upper } \\
\text { quartile }\end{array}$ & $\begin{array}{l}\text { Standard } \\
\text { deviation }\end{array}$ \\
\hline Healthy diet-low cost $(\mathrm{R} \$ 2.48)$ & $\begin{array}{l}\text { Greens, cauliflower, cabbage, Brussels sprouts/ } \\
\text { Pumpkin, carrot/Orange/Broccoli, cucumber, beetroot, } \\
\text { tomato, string beans/Banana/Papaya/Apple/Tangerine/ } \\
\text { Other green vegetables/Savory biscuits }\end{array}$ & 36.4 & $10-58$ & 44.0 & 9.7 \\
\hline Healthy diet-medium cost ( $R \$ 2.84)$ & $\begin{array}{c}\text { Melon, watermelon/Mango, pear, peach/ } \\
\text { Persimmon, grape/Lemon, passion fruit/Pineapple/ } \\
\text { Ice cream/Avocado, guava, kiwi/Liver/Natural juices/Fish }\end{array}$ & 16.1 & $10-53$ & 19.0 & 6.5 \\
\hline Healthy diet-high cost $(\mathrm{R} \$ 5.06)$ & $\begin{array}{l}\text { Almonds, hazelnuts, walnuts, Brazil nuts/Prunes, } \\
\text { raisins/Whole-wheat pasta/Oatmeal, wheat bran/ } \\
\text { Brown sugar/Honey/Rye bread, whole-wheat bread/ } \\
\text { Soy/Red wine/Brown rice }\end{array}$ & 19.4 & $10-51$ & 23.0 & 6.8 \\
\hline Higher-risk diet-low cost ( $\mathrm{R} \$ 2.27)$ & $\begin{array}{c}\text { Home-baked bread/Manioc, sweet potato, corn/ } \\
\text { Beans, lentils/Sugar/Lard/Whole milk/ } \\
\text { Potato/Cream/Pasta/Pork }\end{array}$ & 37.3 & $10-56$ & 43.0 & 8.0 \\
\hline Higher-risk diet-high cost ( $\mathrm{R} \$ 9.91)$ & $\begin{array}{l}\text { Chocolate/Candy, desserts, sweets/Cream/Ham, baloney, } \\
\text { salami, prosciutto/Industrialized mayonnaise/Cheese/ } \\
\text { Fried foods/Hamburger (fast-food)/Sweet biscuits/Cake }\end{array}$ & 30.1 & $10-53$ & 36.0 & 8.6 \\
\hline
\end{tabular}

* Price in Brazilian Real (R\$) in 2004 (R\$ $1.00=$ US\$ 0.329; R\$ $1.00=$ EUR 0.27).

Source: adapted from Alves et al. 17. 
(14 years or more), with higher income, who had never smoked, and who were physically active (Table 2). The HMC dietary pattern was more frequent in white women who were employed and not obese. The HLC pattern was more frequent in women in stable domestic partnerships. The HHC pattern was characteristic of women with higher socioeconomic status (economic classes $\mathrm{A}$ and $\mathrm{B}$, with higher income and education) who were not sedentary (Table 2).

Table 2

Prevalence ratios (PR) and confidence intervals (Cl) for the effect of demographic, socioeconomic, and behavioral variables on the consumption of a healthy diet in women in Southern Brazil $(n=1,026)$.

\begin{tabular}{|c|c|c|c|c|c|c|c|}
\hline \multirow[t]{3}{*}{ Variables } & \multirow[t]{3}{*}{$\mathbf{n}$} & \multicolumn{6}{|c|}{ High consumption of a healthy diet * } \\
\hline & & \multicolumn{2}{|c|}{ Low cost } & \multicolumn{2}{|c|}{ Medium cost } & \multicolumn{2}{|c|}{ High cost } \\
\hline & & $\%$ & PR $(95 \% \mathrm{Cl})$ & $\%$ & PR $(95 \% \mathrm{Cl})$ & $\%$ & PR $(95 \% \mathrm{Cl})$ \\
\hline Age (years) & & & 0.014 ** & & 0.022 ** & & 0.119 ** \\
\hline $20-29$ & 283 & 18.7 & 1.00 & 20.9 & 1.00 & 25.9 & 1.00 \\
\hline $30-39$ & 255 & 26.1 & $1.39(0.97-2.00)$ & 28.5 & $1.36(0.96-1.92)$ & 23.7 & $0.92(0.65-1.29)$ \\
\hline $40-49$ & 302 & 28.8 & $1.54(1.09-2.16)$ & 29.5 & $1.41(1.01-1.96)$ & 26.8 & $1.04(0.76-1.42)$ \\
\hline $50-60$ & 186 & 29.0 & $1.55(1.06-2.26)$ & 31.7 & $1.52(1.06-2.17)$ & 33.9 & $1.31(0.93-1.83)$ \\
\hline Marital status & & & 0.002 & & 0.856 & & 0.268 \\
\hline With partner & 659 & 29.0 & 1.00 & 27.1 & 1.00 & 28.4 & 1.00 \\
\hline Without partner & 367 & 18.9 & $0.65(0.49-0.86)$ & 27.7 & $1.02(0.80-1.31)$ & 24.7 & $0.87(0.67-1.12)$ \\
\hline Skin color & & & 0.171 & & $<0.001$ & & 0.993 \\
\hline White & 860 & 26.3 & 1.00 & 30.6 & 1.00 & 27.1 & 1.00 \\
\hline Non-white & 166 & 20.5 & $0.78(0.54-1.12)$ & 10.2 & $0.33(0.21-0.55)$ & 27.1 & $1.00(0.73-1.38)$ \\
\hline Economic class & & & $<0.001 * *$ & & $<0.001 * \star$ & & $<0.001 * \star$ \\
\hline$A$ and $B$ & 351 & 33.4 & 1.00 & 47.4 & 1.00 & 36.3 & 1.00 \\
\hline C & 404 & 24.1 & $0.72(0.55-0.94)$ & 21.8 & $0.46(0.36-0.60)$ & 22.6 & $0.62(0.48-0.81)$ \\
\hline $\mathrm{D}$ and $\mathrm{E}$ & 268 & 17.2 & $0.51(0.37-0.72)$ & 8.6 & $0.18(0.12-0.28)$ & 21.6 & $0.60(0.44-0.81)$ \\
\hline \multicolumn{8}{|c|}{ Per capita income as multiple of the } \\
\hline minimum wage (quartile) & & & $0.001 * *$ & & $<0.001 * \star$ & & 0.012 ** \\
\hline $4^{\text {th }}$ & 253 & 35.7 & 1.00 & 50.8 & 1.00 & 36.1 & 1.00 \\
\hline 3 rd & 251 & 23.2 & $0.65(0.47-0.90)$ & 28.8 & $0.57(0.42-0.76)$ & 24.8 & $0.69(0.50-0.95)$ \\
\hline 2nd & 257 & 23.0 & $0.64(0.46-0.89)$ & 19.5 & $0.38(0.28-0.53)$ & 21.8 & $0.60(0.43-0.84)$ \\
\hline $1 \mathrm{st}$ & 253 & 19.4 & $0.54(0.38-0.77)$ & 8.7 & $0.17(0.11-0.27)$ & 24.9 & $0.69(0.50-0.95)$ \\
\hline Education (years of study) & & & $<0.001 * *$ & & $<0.001 * *$ & & $0.009 * *$ \\
\hline $14-23$ & 145 & 41.4 & 1.00 & 60.0 & 1.00 & 40.0 & 1.00 \\
\hline $11-13$ & 257 & 25.9 & $0.63(0.44-0.89)$ & 35.3 & $0.59(0.44-0.79)$ & 28.6 & $0.72(0.51-1.01)$ \\
\hline $8-10$ & 169 & 23.7 & $0.57(0.38-0.85)$ & 14.8 & $0.25(0.16-0.38)$ & 20.1 & $0.50(0.33-0.77)$ \\
\hline $5-7$ & 247 & 19.8 & $0.48(0.33-0.70)$ & 21.1 & $0.35(0.25-0.49)$ & 25.5 & $0.64(0.45-0.91)$ \\
\hline $0-4$ & 191 & 22.5 & $0.54(0.37-0.80)$ & 12.6 & $0.21(0.13-0.33)$ & 23.6 & $0.59(0.40-0.87)$ \\
\hline Currently working & & & 0.856 & & 0.002 & & 0.102 \\
\hline Yes & 591 & 25.6 & 1.00 & 31.6 & 1.00 & 24.8 & 1.00 \\
\hline No & 435 & 25.1 & $0.98(0.76-1.25)$ & 21.4 & $0.68(0.53-0.87)$ & 30.2 & $1.22(0.96-1.54)$ \\
\hline Smoking & & & $0.004 * *$ & & $0.005 * *$ & & 0.157 ** \\
\hline Never smoked & 606 & 27.1 & 1.00 & 29.9 & 1.00 & 28.6 & 1.00 \\
\hline Yes, former smoker & 189 & 35.1 & $1.30(0.97-1.73)$ & 30.9 & $1.03(0.77-1.39)$ & 27.7 & $0.97(0.71-1.32)$ \\
\hline Yes, current smoker & 231 & 13.0 & $0.48(0.33-0.71)$ & 17.4 & $0.58(0.41-0.82)$ & 22.6 & $0.79(0.58-1.08)$ \\
\hline Alcohol (grams ethanol/day) & & & 0.394 & & 0.677 & & 0.086 \\
\hline$<30$ & 966 & 25.7 & 1.00 & 27.1 & 1.00 & 26.4 & 1.00 \\
\hline$\geq 30$ & 60 & 20.0 & $0.78(0.44-1.39)$ & 30.0 & $1.11(0.69-1.78)$ & 38.3 & $1.45(0.95-2.23)$ \\
\hline Sedentary & & & 0.024 & & 0.030 & & 0.012 \\
\hline Yes & 703 & 23.0 & 1.00 & 24.9 & 1.00 & 24.3 & 1.00 \\
\hline No & 323 & 30.6 & $1.33(1.04-1.71)$ & 32.5 & $1.31(1.03-1.67)$ & 33.1 & $1.36(1.07-1.74)$ \\
\hline
\end{tabular}

* Results in bold type represent significant associations;

$\star \star$ Linear trend test. 
The RLC pattern showed an inverse association with socioeconomic variables, whereas the RHC pattern showed a trend towards greater consumption among women in better socioeconomic situations. The RLC pattern was characteristic of women with partners, coming from economic classes C, D, and E, with lower income, less education, and who were unemployed (Table 3 ). The RHC pattern was characteristic of younger white women (age 20-29 years) from economic classes

\section{Table 3}

Prevalence ratios (PR) and confidence intervals (CI) for the effect of demographic, socioeconomic, and behavioral variables on the consumption of a higher-risk diet in women in Southern Brazil $(n=1,026)$.

\begin{tabular}{|c|c|c|c|c|c|}
\hline \multirow[t]{3}{*}{ Variables } & \multirow[t]{3}{*}{$\mathbf{n}$} & \multicolumn{4}{|c|}{ High consumption of a higher-risk diet * } \\
\hline & & \multicolumn{2}{|c|}{ Low cost } & \multicolumn{2}{|c|}{ High cost } \\
\hline & & $\%$ & PR $(95 \% \mathrm{Cl})$ & $\%$ & PR $(95 \% \mathrm{Cl})$ \\
\hline Age (years) & & & $0.480 * *$ & & $<0.001 * \star$ \\
\hline $20-29$ & 283 & 22.3 & 1.00 & 42.0 & 1.00 \\
\hline $30-39$ & 255 & 29.6 & $1.33(0.95-1.85)$ & 22.9 & $0.55(0.40-0.75)$ \\
\hline $40-49$ & 302 & 27.8 & $1.25(0.90-1.73)$ & 25.2 & $0.60(0.45-0.80)$ \\
\hline $50-60$ & 186 & 25.3 & $1.13(0.78-1.65)$ & 16.7 & $0.40(0.27-0.59)$ \\
\hline Marital status & & & $<0.001$ & & 0.354 \\
\hline With partner & 659 & 31.6 & 1.00 & 26.6 & 1.00 \\
\hline Without partner & 367 & 16.7 & $0.53(0.40-0.70)$ & 29.8 & $1.12(0.88-1.42)$ \\
\hline Skin color & & & 0.123 & & 0.025 \\
\hline White & 860 & 25.2 & 1.00 & 29.4 & 1.00 \\
\hline Non-white & 166 & 31.9 & $1.27(0.94-1.71)$ & 19.3 & $0.66(0.45-0.95)$ \\
\hline Economic class & & & 0.001 ** & & $<0.001 * \star$ \\
\hline$A$ and $B$ & 351 & 17.1 & 1.00 & 42.3 & 1.00 \\
\hline C & 404 & 31.8 & $1.85(1.36-2.52)$ & 25.6 & $0.60(0.47-0.78)$ \\
\hline$D$ and $E$ & 268 & 29.9 & $1.74(1.25-2.43)$ & 11.9 & $0.28(0.19-0.41)$ \\
\hline \multicolumn{6}{|l|}{ Per capita income as a multiple } \\
\hline of the minimum wage (quartile) & & & $<0.001 * \star$ & & $<0.001 \star \star$ \\
\hline $4^{\text {th }}$ & 253 & 7.1 & 1.00 & 41.7 & 1.00 \\
\hline 3 rd & 251 & 30.8 & $4.31(2.58-7.20)$ & 30.4 & $0.73(0.54-0.98)$ \\
\hline 2nd & 257 & 32.7 & $4.58(2.75-7.61)$ & 24.1 & $0.58(0.42-0.79)$ \\
\hline 1 st & 253 & 35.2 & $4.92(2.97-8.17)$ & 14.6 & $0.35(0.24-0.51)$ \\
\hline Education (years of study) & & & $<0.001^{\star}$ & & $<0.001^{\star}$ \\
\hline $14-23$ & 145 & 6.2 & 1.00 & 48.3 & 1.00 \\
\hline $11-13$ & 257 & 18.8 & $3.03(1.49-6.18)$ & 40.4 & $0.84(0.62-1.13)$ \\
\hline $8-10$ & 169 & 22.5 & $3.62(1.75-7.49)$ & 21.9 & $0.45(0.30-0.68)$ \\
\hline $5-7$ & 247 & 37.3 & $6.00(3.03-11.9)$ & 20.2 & $0.42(0.29-0.60)$ \\
\hline $0-4$ & 191 & 40.3 & $6.50(3.26-13.0)$ & 12.0 & $0.25(0.16-0.40)$ \\
\hline Currently working & & & 0.028 & & 0.005 \\
\hline Yes & 591 & 23.3 & 1.00 & 31.7 & 1.00 \\
\hline No & 435 & 30.4 & $1.31(1.03-1.66)$ & 22.3 & $0.70(0.55-0.90)$ \\
\hline Smoking & & & 0.691 ** & & $0.001 * \star$ \\
\hline Never smoked & 606 & 27.1 & 1.00 & 31.8 & 1.00 \\
\hline Yes, former smoker & 189 & 23.9 & $0.88(0.63-1.23)$ & 27.1 & $0.85(0.63-1.16)$ \\
\hline Yes, current smoker & 231 & 26.1 & $0.96(0.72-1.29)$ & 17.4 & $0.55(0.39-0.77)$ \\
\hline \multicolumn{6}{|l|}{ Alcohol (grams ethanol/day) } \\
\hline$<30$ & 966 & 26.4 & 1.00 & 27.8 & 1.00 \\
\hline$\geq 30$ & 60 & 25.0 & $0.95(0.56-1.60)$ & 26.7 & $0.96(0.58-1.59)$ \\
\hline \multicolumn{6}{|l|}{ Sedentary } \\
\hline Yes & 703 & 27.7 & 1.00 & 27.7 & 1.00 \\
\hline No & 323 & 27.9 & $0.84(0.64-1.09)$ & 27.9 & $1.00(0.78-1.29)$ \\
\hline
\end{tabular}

* Results in bold type represent significant associations;

** Linear trend test; 
$\mathrm{A}$ and $\mathrm{B}$, with higher income and more education (14 years or more), who never smoked, were employed, and who reported no hypertension or were not obese or overweight (Table 3 ).

After multivariate analysis, the frequency of consumption of the diet associated with the HLC pattern was higher in women over 40 years old, who lived with a partner, had a higher level of education (14 years or more), and who had never smoked; the HMC pattern was characteristic of white women over 50 years old, from economic classes A and B, and with a higher level of education (14 years or more); and the HHC pattern was characteristic of women from economic classes A and $\mathrm{B}$, who were not currently working and who were physically active (Table 4$)$.

Table 4

Prevalence ratios (PR) and confidence intervals $(\mathrm{Cl})$ for the effect of demographic, socioeconomic, and behavioral variables on the consumption of a healthy diet in women in Southern Brazil: a multivariate analysis $(n=1,026)$.

\begin{tabular}{|c|c|c|c|}
\hline \multirow[t]{3}{*}{ Block/Variable } & \multicolumn{3}{|c|}{ High consumption of healthy diet * } \\
\hline & Low cost & Medium cost & High cost \\
\hline & PR $(95 \% \mathrm{Cl})$ & PR (95\%Cl) & PR (95\%Cl) \\
\hline \multicolumn{4}{|l|}{ । } \\
\hline Age (years) & $<0.098 * \star$ & $0.093 * \star$ & 0.376 ** \\
\hline $20-29$ & 1.00 & 1.00 & 1.00 \\
\hline $30-39$ & $1.26(0.87-1.83)$ & $1.31(0.92-1.87)$ & $0.90(0.63-1.27)$ \\
\hline $40-49$ & $1.49(1.05-2.11)$ & $1.38(0.98-1.94)$ & $1.00(0.72-1.39)$ \\
\hline $50-60$ & $1.52(1.02-2.26)$ & $1.60(1.10-2.33)$ & $1.24(0.86-1.78)$ \\
\hline Marital status & $<0.001 \star \star$ & $0.438 * \star$ & $0.465 * \star$ \\
\hline With partner & 1.00 & 1.00 & 1.00 \\
\hline Without partner & $0.62(0.47-0.81)$ & $1.11(0.85-1.44)$ & $0.91(0.69-1.18)$ \\
\hline Skin color & 0.691 ** & 0.002 ** & $0.440 * \star$ \\
\hline White & 1.00 & 1.00 & 1.00 \\
\hline Non-white & $0.93(0.64-1.34)$ & $0.47(0.28-0.79)$ & $1.15(0.82-1.61)$ \\
\hline 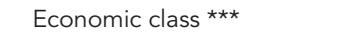 & $0.349 * \star$ & $<0.001 \star \star$ & $<0.001 \star \star$ \\
\hline$A$ and $B$ & 1.00 & 1.00 & 1.00 \\
\hline C & $0.96(0.70-1.32)$ & $0.68(0.50-0.91)$ & $0.62(0.47-0.81)$ \\
\hline$D$ and $E$ & $0.75(0.49-1.15)$ & $0.33(0.20-0.54)$ & $0.57(0.41-0.78)$ \\
\hline Education (years of study) & $0.001 * \star$ & $<0.001$ ** & 0.312 ** \\
\hline $14-23$ & 1.00 & 1.00 & 1.00 \\
\hline $11-13$ & $0.64(0.45-0.90)$ & $0.77(0.57-1.05)$ & $0.80(0.56-1.14)$ \\
\hline 8-10 & $0.55(0.37-0.83)$ & $0.40(0.25-0.64)$ & $0.61(0.38-0.97)$ \\
\hline $5-7$ & $0.45(0.31-0.66)$ & $0.59(0.40-0.88)$ & $0.79(0.52-1.20)$ \\
\hline $0-4$ & $0.51(0.35-0.76)$ & $0.35(0.21-0.60)$ & $0.72(0.45-1.14)$ \\
\hline Currently working & $0.739 * \star$ & $0.246 * \star$ & $0.035 * \star$ \\
\hline Yes & 1.00 & 1.00 & 1.00 \\
\hline No & $1.05(0.81-1.36)$ & $0.86(0.66-1.11)$ & $1.29(1.02-1.64)$ \\
\hline \multicolumn{4}{|l|}{ II } \\
\hline Smoking & $<0.001$ ** & 0.380 ** & $0.463 * \star$ \\
\hline Never smoked & 1.00 & 1.00 & 1.00 \\
\hline Yes, former smoker & $1.24(0.93-1.66)$ & $1.02(0.75-1.38)$ & $0.91(0.67-1.25)$ \\
\hline Yes, current smoker & $0.54(0.36-0.80)$ & $0.79(0.55-1.13)$ & $0.82(0.60-1.14)$ \\
\hline Alcohol (grams ethanol/day) & 0.581 ** & 0.807 ** & $0.163 * \star$ \\
\hline$<30$ & 1.00 & 1.00 & 1.00 \\
\hline$\geq 30$ & $0.85(0.48-1.53)$ & $1.06(0.66-1.72)$ & $1.37(0.90-2.11)$ \\
\hline Sedentary & 0.066 ** & 0.108 ** & $0.019 * \star$ \\
\hline Yes & 1.00 & 1.00 & 1.00 \\
\hline No & $1.27(0.99-1.64)$ & $1.22(0.96-1.56)$ & $1.34(1.05-1.71)$ \\
\hline
\end{tabular}

* Results in bold type represent significant associations;

** $p$ value for likelihood ratio test;

*** Likelihood ratio was tested for both income and socioeconomic class, and class was chosen for its greater significance and to preserve sample size. 
On the other hand, the RLC pattern was characteristic of women living with partners and with lower income and education (7 years or less), and the RHC pattern was characteristic of younger women (20-29 years old) from economic classes $A$ and $B$ who had a higher level of education (14 years or more) (Table 5).

\section{Discussion}

The data in this study revealed the socioeconomic inequalities in food consumption among Brazilian women. Healthy dietary patterns, independent of cost, were more frequent among women with higher income and educational levels. On the other hand, a lower cost, but higher risk diet (i.e. RLC) was more frequently associated with low-income women and with women with fewer years of education. It is especially noteworthy that the RHC pattern was more frequent in younger women, in those from higher socioeconomic classes, and in those with higher levels of education. When interpreting this result, one should consider that the RHC diet was four times more expensive than the low cost diets (Table 1).

Our results are similar to those reported in studies conducted in Brazil and in other countries $6,8,11,22$. Women with better socioeconomic conditions have healthier dietary patterns irrespective of cost. This trend has also been observed for the association between fruit and vegetable consumption and educational levels in a sample of Brazilian adults ${ }^{23}$. In the present study, however, women with similar socioeconomic characteristics were also more frequently associated with the high cost, higher-risk diet (RHC). This is consistent with greater adherence to the Western dietary pattern observed among people with higher education in Spain 24. A previous study reported an association between increased income and education and high consumption of total lipids 5. On the other hand, the higher-risk diet with low cost (RLC) showed an inverse association with education and income. A study of household consumption showed that food group availability is significantly affected by family income ${ }^{9}$. Meat, milk and dairy, fruits and vegetables, alcoholic beverages, condiments, and ready-made meal intake uniformly increases as family income increases. Income is inversely associated with the consumption of rice, beans, roots and tubers, and sugar; there was lower consumption of these foods as income increases. Fats and sweets provide dietary energy at a low cost, while fruits and vegetables provide dietary energy at a much higher cost 23 . The cost analysis from that study showed that every extra $100 \mathrm{~g}$
Table 5

Prevalence ratios (PR) and confidence intervals $(\mathrm{Cl})$ for the effect of demographic, socioeconomic, and behavioral variables on the consumption of a higher-risk diet in women in Southern Brazil: multivariate analysis $(n=1,026)$.

\begin{tabular}{|c|c|c|}
\hline \multirow[t]{3}{*}{ Block/Variable } & \multicolumn{2}{|c|}{ High consumption of higher-risk diet * } \\
\hline & Low cost & High cost \\
\hline & PR (95\%Cl) & PR (95\%Cl) \\
\hline \multicolumn{3}{|l|}{ I } \\
\hline Age (years) & $0.668 * \star$ & $<0.001 * \star$ \\
\hline $20-29$ & 1.00 & 1.00 \\
\hline $30-39$ & $1.17(0.83-1.65)$ & $0.53(0.38-0.73)$ \\
\hline $40-49$ & $1.11(0.79-1.56)$ & $0.59(0.44-0.80)$ \\
\hline $50-60$ & $0.95(0.63-1.43)$ & $0.41(0.27-0.63)$ \\
\hline Marital status & $<0.001$ & 0.481 \\
\hline With partner & 1.00 & 1.00 \\
\hline Without partner & $0.58(0.44-0.78)$ & $0.91(0.70-1.18)$ \\
\hline Skin color & $0.875 * \star$ & 0.293 ** \\
\hline White & 1.00 & 1.00 \\
\hline Non-white & $1.03(0.75-1.40)$ & $0.82(0.56-1.20)$ \\
\hline Economic class *** & & $<0.001 \star \star$ \\
\hline$A$ and $B$ & & 1.00 \\
\hline C & & $0.70(0.52-0.93)$ \\
\hline$D$ and $E$ & & $0.36(0.23-0.57)$ \\
\hline \multicolumn{3}{|l|}{ Per capita income as multiplel } \\
\hline of the minimum wage (quartile) & $<0.001 * \star$ & \\
\hline 4 th & 1.00 & \\
\hline 3 rd & $3.18(1.80-5.61)$ & \\
\hline 2nd & 3.07 (1.72-5.49) & \\
\hline 1 st & $3.17(1.76-5.71)$ & \\
\hline Education (years of study) & 0.020 ** & $0.025 * \star$ \\
\hline $14-23$ & 1.00 & 1.00 \\
\hline $11-13$ & $1.63(0.76-3.51)$ & $0.86(0.62-1.18)$ \\
\hline $8-10$ & $1.55(0.69-3.46)$ & $0.60(0.39-0.92)$ \\
\hline $5-7$ & $2.33(1.07-5.06)$ & $0.68(0.45-1.03)$ \\
\hline $0-4$ & $2.46(1.12-5.41)$ & $0.46(0.27-0.79)$ \\
\hline Currently working & 0.928 ** & $0.409 * \star$ \\
\hline Yes & 1.00 & 1.00 \\
\hline No & $0.99(0.76-1.28)$ & $0.90(0.70-1.16)$ \\
\hline \multicolumn{3}{|l|}{ ॥ } \\
\hline Smoking & $0.636 * *$ & $0.201 * *$ \\
\hline Never smoked & 1.00 & 1.00 \\
\hline Yes, former smoker & $0.88(0.63-1.23)$ & $0.99(0.72-1.36)$ \\
\hline Yes, current smoker & $0.89(0.66-1.21)$ & $0.73(0.51-1.05)$ \\
\hline Alcohol (grams ethanol/day) & $0.860 * \star$ & $0.830 * \star$ \\
\hline$<30$ & 1.00 & 1.00 \\
\hline$\geq 30$ & $1.05(0.61-1.82)$ & $0.95(0.57-1.57)$ \\
\hline Sedentary & 0.523 ** & $0.541 * \star$ \\
\hline Yes & 1.00 & 1.00 \\
\hline No & $0.92(0.70-1.20)$ & $0.92(0.72-1.19)$ \\
\hline
\end{tabular}

* Results in bold type represent significant associations;

** $\mathrm{p}$ value for likelihood ratio test;

$\star \star \star$ Likelihood ratio was tested for both income and socioeconomic class, and class was chosen for its greater significance and to preserve sample size. 
of fats and sweets eaten decreased food costs by between 0.05 and 0.4 Euros, whereas every extra $100 \mathrm{~g}$ of fruit and vegetables eaten increased food costs by between 0.18 and 0.29 Euros.

In our study and in others, age was significantly associated with most of the observed dietary patterns. Women over 40 and 50 years of age were predominantly associated with the low- and medium-cost dietary patterns, respectively. Jaime \& Monteiro 23, in an analysis of fruit and vegetable consumption by Brazilian adults, found a direct association with age. However, younger women (20-29 years) were more likely to be associated with the higher-risk diet-high cost pattern. A British study found a similar association between greater consumption of foods rich in fat among younger subjects ${ }^{12}$. Another study, conducted in Spain, also found an association between younger age and greater consumption of fat-rich diets, which were characterized by high consumption of fast food and other processed products 24 . Such diets had characteristics very similar to the high cost higher-risk diet pattern in our study. Age was also negatively associated with diets rich in fat and meat, and was positively associated with diets rich in fruit and vegetables in the United States ${ }^{8}$. On the other hand, the study by Bonomo et al. 5 reported that greater age was associated with inadequate lipid intake in Brazil.

In our study, consumption by white women fit the healthy diet-medium cost pattern more often than consumption by non-white women. In the study conducted in the United States, the Western pattern, which was considered to be a risk pattern, was associated with non-white skin color 22. On the other hand, in the study conducted in Northeastern and Southeastern Brazil, non-white skin was associated with a traditional diet (with a predominance of rice, beans, flour and sugar) 6 .

In the present study, there was a trend for women with partners to consume lower-cost diets that were either Higher-Risk (RLC) or Healthy (HLC). Whichelow \& Prevost 12 found an association between the consumption of industrialized foods, similar to the higher-risk diet-high cost patterns, and living alone. In the study by Sánchez-Villegas et al. ${ }^{24}$, greater consumption of a Western diet was also more frequent among single subjects.

Regarding smoking, our findings are in agreement with other reports 10,12,22. Women who did not smoke showed greater consumption of healthy diet-medium cost and healthy diet-low cost. Similar results were found in a study in which female former smokers showed low adherence to the Western diet. The authors argue that these results may be due to the fact that women who quit smoking are more likely to make positive decisions with regard to their health, including choosing healthy foods 24 . Nevertheless, smoking was associated with a higher-risk dietary pattern in another study 8 . We also found an association between the higher-risk diet-high cost pattern and people who reported that they had never smoked. However, these associations lost statistical significance after multivariate analysis.

Non-sedentary women showed greater consumption of healthy diets (HLC, HMC, and HHC). Similar results were obtained in other studies $6,8,10,22,24$. However, after multivariate analysis, only the healthy diet-high cost pattern remained significantly associated with physical activity. Moreover, this was the only dietary pattern to maintain a significant association with not working outside the home at the time of the study.

After multivariate analysis, variables that remained strongly associated with dietary patterns were those of a socioeconomic character (income or economic class and education). Associations with demographic and behavioral variables lost their strength after controlling for potential confounders.

Two of our findings stand out. First, despite the similarity in terms of price per serving between the low- and medium-cost healthy patterns (HLC and HMC), there was a large difference in the mean consumption of these diets. This provides evidence of dietary monotony within a homogeneous economic strata of the population. Second, we found that the two higher risk diets had high mean consumption (RLC = 37.3 and $\mathrm{RHC}=30.1$ ). Furthermore, surprisingly, each serving of food for the higher-risk diet-high cost patterns was about four times the price of a serving of the low and medium cost healthy diets (HLC and HMC).

The present study showed that dietary choices of women are not only dependent on food price. Considering the two low-cost diets, healthier and higher-risk diets, wealthier women were more likely to follow a healthier diet than women with lower socioeconomic characteristics, who were more likely to follow a higher-risk diet. Our findings can contribute to policy making processes by highlighting the need for implementing educational interventions and promoting healthy diets at accessible costs to the population; that is making healthy choices easier. 


\section{Resumo}

Para identificar fatores relacionados com padrões alimentares, realizou-se um estudo transversal de base populacional com uma amostra de 1.026 mulheres entre 20 a 60 anos, no Sul do Brasil. Os padrões alimentares foram identificados com análise de componentes principais. Razões de prevalência e intervalos de 95\% de confiança foram calculados com regressão de Poisson. Após ajustamento, os padrões alimentares saudáveis foram mais prevalentes em mulheres com melhor escolaridade e classe econômica. Padrão Saudável de Baixo Custo foi mais prevalente entre mulheres casadas/união e Padrão Saudável de Alto Custo naquelas que não trabalhavam e tinham atividade física regular. Mulheres com baixa escolaridade foram menos prováveis de seguir o Padrão Saudável de Baixo Custo, e sim mais prováveis de seguir Padrão de Risco de Baixo Custo. O Padrão de Risco de Baixo Custo também foi mais prevalente entre as mulheres de baixa renda. Padrões alimentares saudáveis de médio e baixo custo foram positivamente associados com a idade. Nossos resultados evidenciam a desigualdade sócio-econômica na escolha feminina do padrão alimentar, mas não apenas definida pelo preço dos alimentos.

Hábitos Alimentares; Fatores Sócio-Econômicos Mulheres

\section{Contributors}

M. T. A. Olinto coordinated the study and provided orientation for the analysis, data interpretation and article write up. A. Lenz wrote the article. A. L. Alves carried out the research and worked on the data analysis. M. P. Pattussi, J. S. Dias-da-Costa, D. G. Bassani and M. Balbinotti contributed in the study design, data analysis and article write up.

\section{Acknowledgments}

The research was financed by the Foundation for Supporting Research in Rio Grande do Sul State (reference Proad2 $n^{\circ}$. 02/0645-9) and the General Call for submissions from the Brazilian Council for Scientific and Technological Development (CNPq, reference $n^{\circ}$. 473478/2003-0 and no. 308833/2006-6), with support from the São Leopoldo Municipal Health Ministry.

\section{References}

1. Jacques PF, Tucker KL. Are dietary patterns useful for understanding the role of diet in chronic disease? Am J Clin Nutr 2001; 73:1-2.

2. Willett WC. Epidemiology Nutritional. 2nd Ed. New York: Oxford University Press; 1998.

3. Hu FB. Dietary pattern analysis: a new direction in nutritional epidemiology. Curr Opin Lipidol 2002; 13:3-9.

4. Barretto SA, Cyrillo DC. Analysis of household expenditures with food in the city of S. Paulo in the 1990's. Rev Saúde Pública 2001; 35:52-9.

5. Bonomo E, Caiaffa WT, Cesar CC, Lopes AC, LimaCosta MF. Food intake according to socioeconomic and demographic profile: the Bambui Project. Cad Saúde Pública 2003; 19:1461-71.

6. Sichieri R, Castro JF, Moura AS. Factors associated with dietary patterns in the urban Brazilian population. Cad Saúde Pública 2003; 19 Suppl 1:S47-53.

7. Kant AK. Dietary patterns and health outcomes. J Am Diet Assoc 2004; 104:615-35.

8. Park SY, Murphy SP, Wilkens LR, Yamamoto JF, Sharma S, Hankin JH, et al. Dietary patterns using the food guide pyramid groups are associated with sociodemographic and lifestyle factors: The Multiethnic Cohort Study. J Nutr 2005; 135:843-9.
9. Levy-Costa RB, Sichieri R, Pontes NS, Monteiro CA. Household food availability in Brazil: distribution and trends (1974-2003). Rev Saúde Pública 2005; 39:530-40.

10. Hendricks KM, Herbold N, Fung T. Diet and other lifestyle behaviors in young college women. Nutr Res 2004; 24:981-91.

11. Mishra G, Ball K, Arbuckle J, Crawford D. Dietary patterns of Australian adults and their association with socioeconomic status: results from the 1995 National Nutrition Survey. Eur J Clin Nutr 2001; 56:687-93

12. Whichelow MJ, Prevost AT. Dietary patterns and their associations with demographic, lifestyle and health variables in a random sample of British adults. Br J Nutr 1996; 76:17-30.

13. Newby P, Tucker K. Empirically derived eating patterns using factor or cluster analysis: a review. Nutr Rev 2004; 62:177-203.

14. Associação Brasileira de Empresas de Pesquisa. Critério de classificação econômica Brasil. http:// www.abep.org/default.aspx? usaritem =arquivo23 (accessed on 19/Dec/2005). 
15. Secretaria de Políticas de Saúde, Ministério da Saúde. Physical activity and life quality contribution in order to obtain a better healthly lifestyle. Rev Saúde Pública 2002; 36:254-6.

16. Moreira LB, Fuchs FD, Moraes RS, Bredemeier M, Cardozo S, Fuchs SC, et al. Alcoholic beverage consumption and associated factors in Porto Alegre, a Southern Brazilian city: a population-based survey. J Stud Alcohol 1996; 57:253-9.

17. Alves ALS, Olinto MTA, Costa JSD, Bairros FS, Balbinotti MAA. Dietary patterns of adult women living an urban area of Southern Brazil. Rev Saúde Pública 2006; 40:865-73.

18. World Health Organization. Diet, nutrition, and the prevention of chronic diseases. Report of joint WHO/FAO Expert Consultation. Geneva: World Health Organization; 2003. (Technical Report Series, 916).

19. Kushi LH, Meyer KA, Jacobs DR. Cereals, legumes, and chronic disease risk reduction: evidence from epidemiologic studies. Am J Clin Nutr 1999; 70:451S-8S
20. Philippi ST, Latterza AR, Cruz ATR, Ribeiro LC. Food pyramid: a guide to food choices. Rev Nutr 1999; 12:65-80.

21. Victora C, Hutty S, Fuchs S, Olinto MTA. The role of conceptual frameworks in epidemiological analysis: a hierarchical approach. Int J Epidemiol 1997; 26:224-7.

22. Kerver JM, Yang EJ, Bianchini L, Song WO. Dietary patterns associated with risk factors for cardiovascular disease in healthy US adults. Am J Clin Nutr 2003; 78:1103-10.

23. Jaime PC, Monteiro CA. Fruit and vegetable intake by Brazilian adults, 2003. Cad Saúde Pública 2005; 21 Suppl 1:S19-24.

24. Sánchez-Villegas A, Delgado-Rodríguez M, Martinez-Gonzáles M, Irala-Estévez J; Seguimiento Universidad de Navarra Group. Gender, age, sociodemographic and lifestyle factors associated with major dietary patterns in the Spanish Project SUN (Seguimiento Universidad de Navarra). Eur J Clin Nutr 2003; 57:285-92.

Submitted on 10/Mar/2008

Final version resubmitted on $31 / \mathrm{Jul} / 2008$

Approved on 12/Aug/2008 